\title{
Atomic-resolution Imaging and Spectroscopy of Iron Oxide Epitaxial Thin Films
}

A. Carranco-Rodriguez ${ }^{1}$, A. Pofelski ${ }^{1}$, S. Cheng ${ }^{1}$, L. Corbellini ${ }^{2}$, A. Pignolet ${ }^{2}$, P. Longo ${ }^{3}$ and G. A. Botton $^{1}$

${ }^{1}$. Department of Materials Science and Eng., McMaster University, Hamilton, ON, Canada.

2. Centre Énergie, Matériaux et Télécommunications, INRS, Varennes, Québec, Canada.

3. Gatan Inc., Pleasanton, CA.

Epsilon ferrite $\left(\varepsilon-\mathrm{Fe}_{2} \mathrm{O}_{3}\right)$ has attracted attention because of its very high magnetocrystalline anisotropy which provides large coercivity [1]. The epsilon ferrite crystal structure is being characterized by an orthorhombic unit cell with the space group Pna2 $2_{1}$ and is expected to exhibit pyroelectricity [2]. Since $\varepsilon$ $\mathrm{Fe}_{2} \mathrm{O}_{3}$ is considered to be a metastable phase of iron oxide, intermediate between the $\alpha$ and $\gamma$ phases of $\mathrm{Fe}_{2} \mathrm{O}_{3}$, it is not as easy to synthesize as the other $\mathrm{Fe}_{2} \mathrm{O}_{3}$ phases due to the need of a stabilization mechanism, for instance size confinement. For this reason, $\varepsilon$-ferrite has been synthesized, most of the times, in the form of nanoparticles [3].

For most solid-state device applications, thin films are most relevant. The growth of epitaxial thin films of $\varepsilon-\mathrm{Fe}_{2} \mathrm{O}_{3}$ on perovskite substrates by pulsed laser deposition (PLD) has been recently reported [4], where the phase stabilization was accomplished through the epitaxial strain induced by the substrate. An alternative substrate for such epitaxial films, more appropriate for devices than perovskites substrates since it can be grown on silicon [5], is yttrium stabilized zirconia (YSZ). Initially, it has been shown that the growth of $\varepsilon-\mathrm{Fe}_{2} \mathrm{O}_{3}$ can be achieved through the deposition of a $\mathrm{GaFeO}_{3} 50 \mathrm{~nm}$ thick buffer layer on top of YSZ for promoting the $\varepsilon-\mathrm{Fe}_{2} \mathrm{O}_{3}$ growth [6]. Recently, $\varepsilon-\mathrm{Fe}_{2} \mathrm{O}_{3}$ epitaxial films have also been obtained on top of YSZ films, without buffer layer [7]. In order to obtain suitable microstructure, it is necessary to ensure a single-phase $\varepsilon-\mathrm{Fe}_{2} \mathrm{O}_{3}$ and epitaxial films with a unique easy axis, since multi-variant epitaxial growth, as well as polycrystalline growth where the effect of dislocations and grain boundaries can also affect the magnetic anisotropy of this material, tend to make the material more isotropic, therefore severely affecting the distinctive properties of interest of $\varepsilon-\mathrm{Fe}_{2} \mathrm{O}_{3}$, namely its very high magnetic anisotropy.

From inspection of scanning and transmission electron microscopy (STEM-TEM) images, it is possible to deduce a columnar-type growth of the film (Figure 1). STEM and annular dark-field (ADF) imaging, as well as Nano Beam Electron Diffraction (NBED) were used to provide information on the atomic arrangement within the crystalline grains, the relative orientation of the columnar grains and to confirm the presence of different in-plane columnar growth regions perpendicular to the YSZ interface. From the identification of the crystalline grain orientation with NBED patterns, supported by direct high-resolution ADF images and the analysis of the respective diffractograms (Figure 2), different domain orientations, forming sharp interfaces between them and the presence of dark regions, within a $5 \mathrm{~nm}$ band at the film/substrate interface, are identified (Figure 1). Some of the domains have been identified to be different oxide phases (Figure 2) possibly consistent with $\mathrm{Fe}_{3} \mathrm{O}_{4}$. From further NBED data acquired over a $100 \mathrm{~nm}$ line scan it was possible to identify two major growth variants, with their [100] and [010] direction parallel to the observation direction corresponding to domains tilted by $90^{\circ}$ with respect to each other. Further electron energy loss spectroscopy (EELS) measurements of the $\mathrm{Fe}_{2,3}$ edge, showed a change in the shape of the spectrum, concomitant with a shift in the onset of the edge (Figure 3), consistent with the presence of different iron oxide phases at the interface with a lower average valence state. The existence of different 
grain orientations and a secondary phase in the film, confirmed by the changes observed in EELS spectrum, are most certainly factors contributing to the reported pinched hysteresis loop $[7,8]$.

References:

[1] M. Yoshikiyo et al, J. Phys. Chem. C 15 (2012), p. 8688.

[2] S. Ohkoshi et al, Sci Rep. 5 (2015). p. 14414.

[3] J. Jin et al, Adv. Mater. 16 (2004), p. 48.

[4] M. Gich et al, Appl. Phys. Lett. 96 (2010), p. 112508.

[5] S. Jun et al, Appl. Phys. Lett. 78 (2001), p. 22.

[6] T. M. N. et al, J. Appl. Phys. 120 (2016), p. 185304.

[7] L. Corbellini et al, Sci Rep. 7 (2017), p. 3712.

[8] We are pleased to acknowledge NSERC funding for supporting for this work.
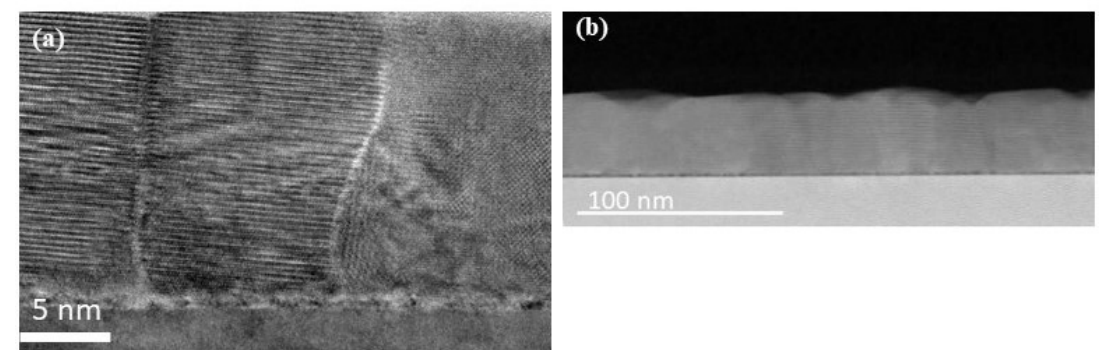

Figure 1. (a) High Resolution TEM image showing the boundaries between columnar structures. (b) High Resolution STEM image of a $50 \mathrm{~nm}$ thick $\varepsilon-\mathrm{Fe}_{2} \mathrm{O}_{3}$ directly on YSZ (100) substrate showing columnar-type growth along the film.
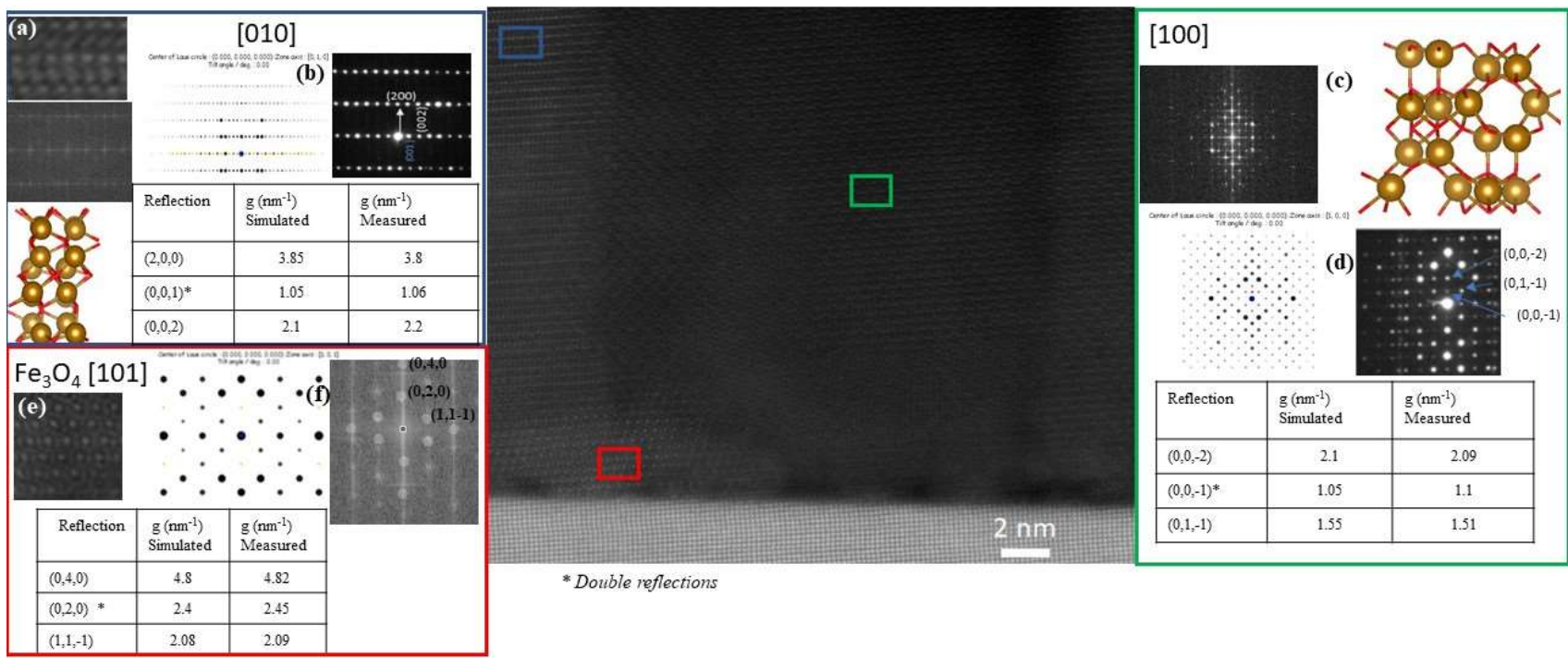

* Double reflections

Figure 2. High resolution STEM image of cross-section $\varepsilon-\mathrm{Fe}_{2} \mathrm{O}_{3}$ on YSZ (100) substrate including detailed image, diffractogram and atomic models; ( $a, b)$ NBED pattern and simulated pattern of the [010] zone axis; (c-d) NBED pattern and simulated pattern (d) of [100] zone axis; (e) Detailed higher magnification image of the $\mathrm{Fe}_{3} \mathrm{O}_{4}$ phase (red inset) with its (f) simulated pattern and diffractogram.

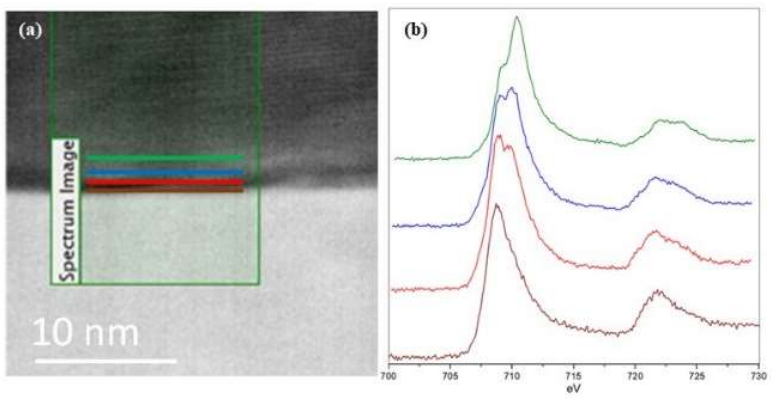

Figure 3. (a) Area selected for the EELS analysis with regions identified with colored regions. (b) $\mathrm{FeL}_{2,3}$ spectra acquired over the regions identified with color coded plots corresponding to the areas shown in (a). 